Estudios Constitucionales, Año 14, No 1, 2016, pp. 291-306

ISSN 07180195

Centro de Estudios Constitucionales de Chile Universidad de Talca

"La regulación del referéndum de especial trascendencia en perspectiva comparada

y su eventual reconocimiento en la Constitución chilena"

Francisco Soto Barrientos

\title{
LA REGULACIÓN DEL REFERÉNDUM DE ESPECIAL TRASCENDENCIA EN PERSPECTIVA COMPARADA Y SU EVENTUAL RECONOCIMIENTO EN LA CONSTITUCIÓN CHILENA•
}

\author{
The Regulation of Special Significance Referendum \\ and its Possible Incorporation to the Chilean Constitution
}

\author{
Francisco Soto BarRientos • \\ Universidad de Chile \\ fsoto@derecho.uchile.cl
}

RESUMEN: El referéndum es una institución frecuentemente regulada por las constituciones de los Estados. Este trabajo indaga sobre un particular tipo: el referéndum de especial trascendencia. Aunque sólo en un acotado grupo de cartas se establece, esta fórmula ha cobrado notoriedad al ser utilizada por Grecia (5 de julio de 2015) para consultar sobre la aplicación de medidas de ajuste económico exigidas como condición para permanecer en la Eurozona. La incorporación de esta institución en la Constitución chilena es vista por un número importante de parlamentarios y organizaciones de la sociedad civil como la alternativa más idónea para que la ciudadanía pueda decidir el mecanismo para cambiar la actual Constitución. En primer lugar, analizaremos la experiencia comparada sobre referéndum, para luego realizar un análisis pormenorizado de los referéndums de especial trascendencia. Finalmente, nos detendremos a sistematizar los problemas constitucionales que tendría la aprobación de esta iniciativa, particularmente referida a una eventual consulta sobre el mecanismo de remplazo constitucional.

ABSTRACT: The referendum is an institution commonly included in the States' constitutions. This paper is about one particular kind: the special significance referendum. Even though the former is regulated in only a few charters, this type of referendum has become well-known since its application by Greek authorities on July 5th, 2015 to seek the public's opinion over the application of policies of economic adjustment demanded as a condition to remain in the Eurozone. The incorporation of this institution to the Chilean constitution is regarded by a considerable number of congressmen and civil

\footnotetext{
- Trabajo recibido el 18 de agosto de 2015 y aprobado el 20 de enero de 2016. Este trabajo se realiza en el marco de su labor en el Grupo de Estudios sobre Democracia y Constitucionalismo (Gedeco) y del proyecto investigación "Interacción entre representación y participación en la producción normativa" (DER201568160-C3-3-P).

- Licenciado en Ciencias Jurídicas y Sociales, Magíster en Derecho Constitucional de la Universidad Diego Portales y Doctor en Derecho Universidad de Barcelona. Actualmente se desempeña como Profesor Asociado del Departamento de Derecho Público de la Facultad de Derecho de la Universidad de Chile, donde imparte cursos de derecho constitucional y ciencias políticas. Integra el Comité Académico del Programa de Doctorado de dicha facultad, dictando cursos de derecho público y comparado.
} 
society groups as the best alternative choice in order that citizens may decide over the mechanism to change the current constitution. To begin with, we will analyze the comparative experience over the referendum, and then we will throughly analyze the special significance referendums. Finally, we will sistematize the consitutional problems that the approval of this motion may face, in particular, referred to the possible referendum over the mechanism for constitutional replacement.

PALABRAS CLAVE: Referéndum, remplazo constitucional, derecho comparado.

KEYWORDS: Referendum, constitutional replacement, comparative law.

\section{ANTECEDENTES COMPARADOS Y DOCTRINALES:}

LOS DISTINTOS TIPOS DE REFERÉNDUM

El reconocimiento constitucional del referéndum es un fenómeno moderno, vinculado a la democracia representativa y al constitucionalismo. En la polis griega o en las prácticas locales medievales, cuando se querían adoptar decisiones de importancia y en consulta a la ciudadanía, el mecanismo participativo no era el referéndum, sino la asamblea ${ }^{1}$.

Aunque identificamos cierta crítica a su inserción en los sistemas democráticos representativos ${ }^{2}$, hoy en día en la mayoría de las constituciones occidentales se suele regular la figura del referéndum. No obstante, las diferencias respecto de quién cuenta con la iniciativa para realizar la consulta al cuerpo electoral, su carácter obligatorio o consultivo, la jerarquía de la norma a reformar y las materias consultadas, ha generado que no se pueda hablar de un mecanismo, sino de distintos tipos de referéndums.

La doctrina europea identifica al referéndum con consultas vinculadas a actos de naturaleza normativa, mientras que el plebiscito se refiere a cuestiones de hecho, actos políticos y medidas de gobierno. Lo que se regula en las constituciones son los referéndums y no los plebiscitos ${ }^{3}$. En contraste, en las constituciones latinoamericanas, particularmente en Chile, los términos "plebiscito" y "referéndum" son utilizados como sinónimos. La excepción la establece la constitución uruguaya, que distingue el término plebiscito para decidir si aprueba o no un proyecto de reforma constitucional. Mientras con la voz referéndum se regulan materias de rango legal ${ }^{4}$.

Sin ánimo de ser exhaustivos, identificaremos varias fórmulas referendarias, lo que nos permitirá situar al de especial trascendencia en el contexto comparado.

1 LuCIANi (2008), pp. 157-182.

2 SChumpeter (1984), pp. 321 y ss., y SARTORi (1987), pp. 155 y ss.

3 García-Pelayo (1984), pp. 183-185.

4 Soto (2013) y GonZÁlez (2008). 
La primera de las modalidades, la más antigua y utilizada, tiene que ver con la consulta obligatoria frente a una reforma constitucional. Nos encontramos ante una fórmula que dificulta la posibilidad de cambio, ya que no sólo se debe contar con el quórum necesario de parlamentarios, muchas veces agravado, sino que con una opinión pública sensibilizada con la reforma en cuestión y con la voluntad de concurrir a las urnas. En este sentido, el referéndum puede ser considerado como un mecanismo funcional a la estabilidad o conservadurismo constitucional.

Este tipo de referéndum nace con la Constitución suiza de 1848, manteniéndose hasta la fecha en su actual artículo 140. También ha sido recogido por otras cartas europeas como la española (art. 168.2 y 3), la francesa (art. 89), la irlandesa (art. 47), la danesa (art. 88) y la italiana (art. 138). Esta última, con la particularidad de que establece la posibilidad de llamar a referéndum mediante iniciativa popular cuando la reforma no ha logrado alcanzar los dos tercios de apoyo parlamentario. Dicho instrumento ha sido calificado como referéndum facultativo, no obstante que su convocatoria no es controlada por los órganos que participan en la reforma, sino por la ciudadanía 5 .

En Latinoamérica, la Constitución uruguaya también establece referéndum obligatorio en materia constitucional (art. 331, letras a y b), de la misma manera que la boliviana (art. 411.1) y la peruana (art. 206). La Carta fundamental colombiana de 1991 señala en su art. 377 una particular fórmula, ya que restringe su carácter obligatorio para determinadas materias como los derechos fundamentales, los procedimientos de participación popular o las materias requeridas por iniciativa popular.

La segunda de las modalidades referendarias dice relación con aquellas que intervienen en el ámbito legislativo. Éstos son mecanismos que buscan compatibilidad entre democracia representativa y directa. La experiencia constitucional comparada ha justificado explícitamente la incorporación de esta fórmula para dinamizar la acción legislativa a través de la opinión de la ciudadanía. El precursor de este tipo de referéndum fue Kelsen, quien busco revertir las críticas al parlamentarismo y a los partidos políticos a través de la utilización de mecanismos de democracia directa que pudieran insertarse coherentemente en la actividad política, donde los partidos tuviesen un rol protagónico. Para ello identificó que el espacio preferente de participación debía situarse en el Congreso, particularmente en el proceso de elaboración de la ley ${ }^{6}$.

5 Olivetti (2007), pp. 157-175.

6 Kelsen (1988), p. 355. 
Desde esta perspectiva, nos encontramos con sistemas que entregan la regulación del referéndum a la ley como es el caso de la Constitución de Suecia (arts. $4^{\circ}$ y 15) o la Carta de Hungría (art. 28/B), que sitúa el referéndum dentro de las competencias del Parlamento.

El referéndum en estos términos ha sido concebido como un instrumento para las minorías parlamentarias. Así, la Constitución de Dinamarca (art. 42) entrega la posibilidad de referéndum facultativo a la minoría parlamentaria dentro del ámbito de la ley. En Italia (art. 75), también busca la participación de la minoría política, pero de una singular manera: el referéndum abrogativo. Esta modalidad de referéndum permite derogar de manera total o parcial una ley o un acto con fuerza de ley, cuando así lo soliciten 500 mil electores o cinco Consejos Regionales. En el caso particular del referéndum abrogativo italiano, organizaciones sociales y partidos políticos lo han promovido para doblegar leyes establecidas por la mayoría parlamentaria transformándolo en un contrapeso notable a la función legislativa ejercida por el Parlamento. Las principales materias que se han consultado en Italia son: ley de divorcio, impedir la instalación de misiles nucleares en Italia, cambios en el sistema electoral, centrales nucleares, privatización del agua, entre otras ${ }^{7}$.

El referéndum abrogatorio se ha trasplantado a varias constituciones latinoamericanas recientemente: Uruguay (art. 79), Colombia (art. 170), Venezuela (art. 74) y Costa Rica (art. 105).

Finalmente, encontramos en la experiencia comparada los referéndums para ratificar acuerdos que comprometan a la forma de Estado. Esto es, aquellos acuerdos que alteran o modifican la configuración del Estado en el territorio y/o la relación entre los ciudadanos y los poderes públicos ${ }^{8}$. Se trata de consultas obligatorias y vinculantes, cuya convocatoria se encuentra descrita detalladamente en los textos constitucionales. Particularmente, el proceso de integración europeo ha promovido su proliferación. Hoy lo encontramos en constituciones como la de Francia (art. 88.5) Bulgaria (arts. 84.5, 98.1 y 102.3.6), República Checa (art. 62.1) y Dinamarca (art. 19), entre otras.

En Latinoamérica, la Constitución de Costa Rica (art. 168) establece la consulta al cuerpo electoral para decidir sobre la creación de provincias, o bien, el aumento o disminución de su territorio. También, la Carta de Brasil (art. 231 No 5) lo establece para la creación de nuevas unidades de la federación.

7 LuCiani (2005).

8 Pizzorusso (19879, p. 103. 


\section{REFERÉNDUM DE ESPECIAL TRASCENDENCIA}

El referéndum facultativo frente a decisiones de especial trascendencia se encuentra regulado en diversas constituciones de Europa, como España (art. 92), Austria (art. 49b) y Grecia (art. 44). En Latinoamérica, se ha trasplantado esta figura a las cartas de Colombia (art. 104), Guatemala (art. 173), Venezuela (art. 71) y Ecuador (art. 104). Se trata de un referéndum consultivo, que permite orientar definiciones posteriores de los órganos constitucionales y que no necesariamente compromete al Estado con la decisión adoptada.

La característica más común de esta fórmula es que tanto la iniciativa como la definición de lo que se consulta conforman una atribución del jefe de Gobierno o Presidente de la República, quien requerirá el acuerdo del Congreso. Aunque también existen fórmulas alternativas como las que encontramos en Austria y Guatemala, que permiten al Congreso convocar a referéndum de especial trascendencia, o en Venezuela, quizás el modelo más singular, que permite la posibilidad de iniciativa popular, además de extender este mecanismo al ámbito local.

Una primera aproximación doctrinal la encontramos ante la definición de especial trascendencia. Por regla general los textos constitucionales entregan esta definición a la discrecionalidad de la autoridad política. Hans Peter Schneider enfatiza el carácter excepcional de los referéndums restringiéndolo a materias que los parlamentarios (elegidos por un período limitado de tiempo) no tienen la capacidad ni están legitimados para decidir. Este tipo de materias tendrían en común la afectación a generaciones futuras, sobre las cuales no se puede anticipar algún tipo de representación parlamentaria. De esta forma, para Schneider, las consultas directas se deberían concentrar en decisiones que el autor llama "de nuevo tipo" y que clasifica en tres categorías: (a) de riesgos incalculablemente altos; (b) de consecuencias irreversibles para el desarrollo de la humanidad, y (c) de repercusiones internacionales no localizadas y no controlables. El elemento diferenciador sería justamente identificar las materias que caben en algunos de los tipos descritos por Schneider para ser consultadas directamente, distinguiéndolas de las materias que podrían ser sometidas a un debate parlamentario?.

En este sentido, cada carta fundamental debiera fijar las materias que considera de especial trascendencia, o bien establecer un catálogo de materias a consultar obligatoriamente. Sin embargo, como veremos, un análisis pormenorizado de los casos en que se regula el referéndum de especial trascendencia demuestra

9 SChNeider (1991), pp. 262 y ss. 
que esto no ocurre. A continuación, analizaremos quién cuenta con la iniciativa para articular este tipo de consultas y las materias que pueden ser sometidas a la opinión de la ciudadanía.

Sobre la iniciativa de consulta, en España la convocatoria del referéndum de especial trascendencia la realiza el Rey, previa aprobación del Presidente del Gobierno, debatida en el Congreso de Diputados ${ }^{10}$. En Austria lo debe acordar el jefe de Gobierno a través de una propuesta del Gobierno Federal, previa deliberación de la Comisión Principal (instancia represententativa del Congreso), o bien la iniciativa la puede presentar la propia Comisión antes citada.

En el caso de Grecia, lo propone el Presidente de la República con acuerdo del Consejo de Ministros, pero si el tema a consultar tiene que ver con un proyecto de ley que se está discutiendo en el Congreso, la convocatoria a referéndum deberá contar con el apoyo dos quintas partes de los diputados.

En Latinoamérica, las constituciones establecen procedimientos similares. En Colombia, la convocatoria para el referéndum de especial trascendencia supone la iniciativa del Presidente de la República con la firma de todos los ministros y previo pronunciamiento favorable del Senado de la República. En Venezuela, el mecanismo es similar, con la diferencia de que requiere el voto conforme de la mayoría de la Asamblea Nacional. La novedad, como anticipamos, radica en la posibilidad de iniciar el proceso mediante iniciativa popular, a solicitud de un número no menor al 10\% de los electores inscritos en el Registro Civil. Además, en el mismo artículo 71, la Constitución de Venezuela contempla otra modalidad de referéndum para materias de especial trascendencia, pero a nivel local. La iniciativa le corresponde, en este caso, a la Junta Parroquial, al Concejo Municipal y al Consejo Legislativo, por acuerdo de las dos terceras partes de sus integrantes; al alcalde y al Gobernador de Estado o a solicitud de un número no menor del $10 \%$ del total de inscritos en la circunscripción correspondiente.

Las otras dos regulaciones latinoamericanas que contemplan el referéndum de especial trascendencia establecen un procedimiento distinto de iniciativa. En

10 El artículo 6º de la Ley Orgánica 2/1980 sobre distintas modalidades de referéndum señala que en el caso de requerir autorización para llamar a referéndum de especial trascendencia, se requiere el voto conforme de la mayoría absoluta de los diputados sobre la solicitud del Presidente de Gobierno, y que la propuesta sea aprobada por la Cámara en los términos exactos en los que será sometida a la ciudadanía. Una vez que se cuente con dicha aprobación, la convocatoria es efectuada por el Rey mediante Real Decreto acordado en el Consejo de Ministros, con refrendo del Presidente del Gobierno (artículo 2.3 de la LO 2/1980). La fecha de convocatoria no podrá extenderse entre los 30 y 120 días posteriores a la publicación del Real Decreto de convocatoria (artículo 3 o de la LO 2/1980). 
Guatemala se faculta indistintamente al Presidente de la República o al Congreso de la República para convocar consultas sobre materias de especial trascendencia. En tal caso, la autoridad que asume la iniciativa debe fijar con precisión la o las preguntas que se someterán a consulta de los ciudadanos y no requiere buscar el acuerdo con otros órganos constitucionales. Igual cosa sucede con la fórmula prevista en el artículo 104 de la Constitución de Ecuador, que faculta al Presidente de la República a solicitar al Consejo Nacional Electoral que convoque a consulta popular sobre los asuntos que estime convenientes.

En definitiva, de esta breve exposición de la experiencia comparada se colige que nos encontramos ante un mecanismo que busca dar expresión a la mayoría electoral que cuenta con el gobierno y el parlamento. Sólo Austria, Guatemala y Ecuador permiten activar este mecanismo al gobierno o al parlamento, facultando al electorado a través de la consulta resolver eventuales diferencias entre la mayoría parlamentaria y el gobernante.

Sobre las materias consultadas, en la mayoría de las experiencias analizadas nos encontramos con que el referéndum de especial trascendencia acompaña la regulación del referéndum constitucional, del referéndum abrogatorio (que opera en el ámbito legal), entre otros; restringiéndolo a las potestades propias del gobierno. Así, el artículo 49b de la Constitución de Austria dispone que: "Se consultará al electorado sobre una materia determinada de importancia fundamental para toda Austria y cuya regulación sea competencia del Gobierno Federal”, estableciendo como límite la fecha de elecciones y las materias sobre las cuales deba pronunciarse un tribunal o un órgano administrativo.

En España, esto no se define claramente en la Constitución y ha sido un tema debatido por la doctrina. Algunos vinculan este referéndum al proceso de formación de la ley, debido a su ubicación en el capítulo II, del título III de la Constitución española (relativo a "la elaboración de las leyes"). No obstante, la posición mayoritaria de los autores considera que esta fórmula se estableció únicamente para decisiones calificadas por la misma autoridad competente como "de especial trascendencia" y no podría en ningún caso suplantar el normal ejercicio de los poderes constituidos. Por tanto, las materias consultadas no podrían afectar el ámbito legal, restringiéndose al terreno de la potestad reglamentaria del gobierno ${ }^{11}$. López considera que, en el caso español, la negación de ámbito legislativo para este tipo de mecanismo, lo restringe a una "opción política de

11 Aguiar de Luque (2001), p. 128 y Pérez Sola (1994). 
carácter básico", que podría eventualmente dar pie a soluciones legislativas o gubernamentales posteriores ${ }^{12}$. En la práctica, el referéndum facultativo del artículo 92 de la referida Constitución ha sido utilizado sólo en dos ocasiones hasta la fecha: la convocatoria para establecer la permanencia de España en la OTAN y la aprobación del Tratado Constitucional que establece una Nueva Constitución para Europa ${ }^{13}$.

En el caso de Grecia, como ya hemos referido, la propia Constitución en el artículo 44 permite consultar materias que están siendo debatidas en el parlamento, cuando la consulta sea asociada "con un problema social grave". Por tanto, claramente extiende la competencia del referéndum de especial trascendencia al ámbito legal, estableciéndose como límite los proyectos de ley de carácter tributario y los que disponga el Reglamento de la Cámara.

Las constituciones de Austria (art. 141) y Grecia (art. 100.1.b) le entregan amplias facultades a los tribunales constitucionales respectivos para que velen por la constitucionalidad de estos procesos de consulta. España, por su parte, no considera la intervención de este tribunal, sino de la justicia electoral y regula las eventuales reclamaciones que surjan de este proceso en la Ley Orgánica sobre las Distintas Modalidades de Referéndum 2/1980.

Las constituciones latinoamericanas no hacen referencia a las materias que deberán ser consultadas. En Colombia, la ley 134/1994 del 31 de mayo, que regula los mecanismos de participación ciudadana, establece en su artículo 50 que no se podrán realizar consultas sobre temas que impliquen modificación de la Constitución Política, mandatando al tribunal contencioso administrativo para que se pronuncie sobre su constitucionalidad (artículo 52 de la ley 134/1994). El artículo 51 de esta ley precisa que no podrá ser objeto de consulta popular la convocatoria a una Asamblea Constituyente.

Un elemento adicional que puede darnos luces sobre las materias a consultar viene dado por el efecto del referéndum de especial trascendencia. Se trata de un mecanismo que tiene carácter consultivo. Vale decir, su resultado tiene por objetivo orientar la decisión de los órganos constitucionales y no necesariamente imponerles una determinación popular. Esta idea ha sido debatida por la doctrina española, ya que parece impensable que las autoridades tomen definiciones

12 LÓPEZ (2006), p. 215.

13 Welp (2009), pp. 39-53. 
contrarias a la voluntad popular mayoritariamente manifestada ${ }^{14}$. López aclara que consultivo no significa necesariamente no vinculante. Para este autor, este tipo de referéndum tiene un carácter ratificatorio de una decisión prefijada por la autoridad, impidiendo en caso de resultado negativo que ésta pueda ser adoptada. A reglón seguido, López constata que si esto ocurre así, lo consultado sólo puede referirse a materias propias del ámbito gubernamental, ya que como éste posee la iniciativa de convocatoria y determinación de la pregunta, no podría comprometerse con ello la voluntad de otro órgano constitucional ${ }^{15}$.

Esta reflexión podría ser extensiva en varios de los Estados analizados pero claramente no operaría en Grecia, Guatemala y Venezuela, que extienden la posibilidad de presentar iniciativa al Congreso, y en el caso de Venezuela permite inclusive la iniciativa popular. Es más, Grecia extiende la figura a materias de ley expresamente.

La heterogeneidad expuesta hasta acá, nos hace preguntarnos si todas estas figuras, a pesar de hacer referencia a consultas en materia de especial trascendencia, pueden ser asimiladas bajo el mismo rótulo. Con todo, sigue superviviendo un elemento común en todas estas regulaciones, y es que este mecanismo no se extiende al ámbito constitucional, ya sea porque expresamente se señala, o bien porque al discutirse su extensión al nivel legal sería impensable llevarlo a un nivel de mayor jerarquía.

\section{LA EXPERIENCIA CHILENA Y LA MOCIÓN QUE PERSIGUE}

INCORPORAR EL REFERÉNDUM DE ESPECIAL TRASCENDENCIA.

Desde las elecciones presidenciales de 2009 se vienen sucediendo diversas propuestas para cambiar la actual Constitución. Particularmente, los partidos de la Nueva Mayoría -actual coalición gobernante en Chile- han elaborado diversas definiciones programáticas en materia constitucional ${ }^{16}$, propuestas que finalmente

14 Cruz Villalón (1980), pp. 145-168, y Aragón (1989), pp. 115-132.

15 LÓPEZ (2006), p. 217.

16 Disponible se encuentran en la web: Estatuto Programático del Partido por la Democracia: http://www. ppd.cl/wp-content/files_mf/estatutoprogramatico.pdf; Bases Programáticas del PDC, aportes para un debate: http://issuu.com/pdcdigital/docs/bases_programaticas_pdc/1?e=2367133/2879972

Programa del Partido Radical de Chile, Programa para un Chile Digno, disponible en: http://www.partidoradical.cl/v1/wp-content/uploads/2011/06/PROGRAMA-PARA-UN-CHILE-DIGNO.pdf

Propuestas Programáticas del Partido Comunista de Chile para una Convergencia Social y Política: http:// www.pcchile.cl/?page_id=177\&did=12 
fueron sistematizadas en el programa de gobierno de la actual Presidenta, Michelle Bachelet ${ }^{17}$.

Paralelo al debate de contenido se ha desarrollado una acalorada discusión sobre el procedimiento para cambiar la actual Constitución. Esto básicamente por los altos quórums que el actual procedimiento de reforma impone, que exigen entre un 60\% y 66\% de los diputados y senadores en ejercicio (art. 127 de la Constitución), formulándose numerosas alternativas al respecto, las que hemos analizado sistemáticamente en otro trabajo ${ }^{18}$. Sobre este punto cabe señalar que el programa de Michelle Bachelet sólo señala que el proceso de elaboración de una nueva constitución deberá reunir tres características: democrática, institucional y participativo ${ }^{19}$.

En ese contexto, se instala en el debate público la propuesta de realizar un plebiscito que permita a la ciudadanía optar por el mecanismo para elaborar una nueva carta fundamental ${ }^{20}$. El 15 de abril de 2015 se presentó una moción tendiente a incorporar el plebiscito de especial trascendencia mediante la reforma de los artículos 15 y 32 No 4 de la Constitución, patrocinada por un grupo de parlamentarios que se autodenominan "Bancada Transversal de Diputados y Diputadas por una Asamblea Constituyente" 21 y por veinticinco constitucionalistas que trabajan en el movimiento "Marca $\mathrm{AC}^{22}$. En el presente apartado de este trabajo realizaremos un análisis crítico de esta iniciativa.

La reforma propuesta persigue modificar el carácter acotado que tiene el plebiscito nacional en la Constitución de 1980. Esta fórmula no opera frente a cualquier materia de relevancia nacional que la ciudadanía o que algún poder del Estado quiera promover, sino que se establece frente a un supuesto de conflictos entre los órganos colegisladores durante el proceso de tramitación de una reforma

17 Programa de Gobierno Michelle Bachelet 2014-2018, p. 34: http://michellebachelet.cl/programa/.

18 Sото (2014), pp. 397-427.

19 Programa de Gobierno Michelle Bachelet 2014-2018, p. 35: http://michellebachelet.cl/programa/.

20 Garretón (2014), p. 4.

21 Compuesta por los diputados: Sergio Aguiló, Jenny Álvarez, Claudio Arriagada, Gabriel Boric, Karol Cariola, Lautaro Carmona, Daniella Cicardini, Maya Fernández, Iván Fuentes, Cristina Girardi, Hugo Gutiérrez, Marcela Hernando, Jorge Insunza, Giorgio Jackson, Luis Lemus, Daniel Melo, Vlado Mirosevic, Manuel Monsalve, Daniel Núñez, Clemira Pacheco, Denise Pascal, Roberto Poblete, Yasna Provoste, Gaspar Rivas, Alberto Robles, Luis Rocafull, Raúl Saldívar, Leonardo Soto, Guillermo Teillier y Camila Vallejo.

22 Detalles de la señalada iniciativa pueden ser revisados en el sitio web: http://www.marcaac.cl/site/. 
constitucional. Nos encontramos frente a un supuesto de conflicto que nunca se ha dado y de difícil operatoria.

En Chile, en los últimos cuarenta años, en tres momentos se ha consultado de manera directa al cuerpo electoral y en todas estas ocasiones el fundamento jurídico fueron normas transitorias para: (i) aprobar la nueva Constitución (septiembre del 1980), (ii) definir la continuidad del gobierno del general Augusto Pinochet (octubre de 1988) y (iii) establecer modificaciones mínimas que permitirían dar inicio a la transición democrática (junio de 1989)23.

En síntesis, la moción parlamentaria en comento busca hacer operativa la alternativa de consulta directa a la ciudadanía, entregándole al Presidente de la República con acuerdo del Congreso la iniciativa para llamar a un plebiscito nacional de especial trascendencia, junto con facultar al legislador para que pueda establecer nuevos mecanismos de consulta.

Según la Constitución vigente en Chile, sólo puede llamarse a plebiscito en los casos previstos expresamente en dicha norma fundamental (art. $5^{\circ}$ y 15 inciso $\left.2^{\circ}\right)$. El Tribunal Constitucional ha ratificado este criterio de interpretación en sentencias No 279/1998, del 6 de octubre, y No 1050/2008, del 10 de marzo.

El proyecto propone modificar el inciso segundo del artículo 15, agregando antes de su punto final la expresión " $y$ las leyes". De prosperar la reforma, el eventual texto quedaría de la siguiente manera: "Sólo podrá convocarse a votación popular para las elecciones y plebiscitos expresamente previstos en esta Constitución y las leyes."

Adicionalmente, se establece modificar la facultad del Presidente de la República para llamar a plebiscito, reemplazando el actual artículo 32 No 4 por el siguiente: "Convocar a plebiscito en los casos en que corresponda conforme a la Constitución y a las leyes. El Presidente podrá convocar en todo caso a plebiscito si cuenta para ello con el acuerdo de ambas cámaras del Congreso Nacional".

Con todo, el procedimiento para llamar a plebiscito seguiría siendo el mismo. En este sentido, el artículo 129 de la Constitución establece que la convocatoria se realizará mediante decreto supremo que fijará la fecha de la votación plebiscitaria, la que no podrá tener lugar antes de treinta días ni después de sesenta, contados desde la publicación de dicho decreto.

Dicho decreto se encuentra sometido a control de constitucionalidad por el Tribunal Constitucional. La referencia expresa se encuentra en el 93 No 5

23 Soto (2013), p. 227. 
y es un control facultativo que debe promoverse a requerimiento del Senado o de la Cámara de Diputados, dentro de 10 días contados desde la fecha de publicación del decreto que fije el día de la consulta plebiscitaria (art. 93 inciso $8^{\circ}$ ). Según la Ley Orgánica del Congreso (art. 78 inciso 2o), esta impugnación debe fundamentarse en aspectos específicos: procedencia y oportunidad. En este caso, nos encontraríamos ante un plebiscito que cuenta con el respaldo del Congreso, por lo que no sería lógico que el Congreso requiriera la participación del Tribunal Constitucional.

No obstante, quedaría una alternativa de intervención de este Tribunal. Nos referimos a la hipótesis establecida en el artículo 93 No 9, vale decir, que la inconstitucionalidad del decreto que convoque a plebiscito sea establecida por la Contraloría General de la República. En este caso, es el Presidente de la República quien debe remitir los antecedentes al Tribunal Constitucional para salvar la representación de la Contraloría, ya que no tiene la facultad de insistir según el artículo 99 inciso $3^{\circ}$.

El tema del control de constitucionalidad tiene importancia debido a que la moción persigue determinar el procedimiento de cambio constitucional. La estrategia declarada por los parlamentarios y organizaciones que la sustentan es justamente consultar a la ciudadanía por el mecanismo pertinente para el cambio constitucional. Para esto, se espera contar con la complicidad del gobierno, que debería iniciar este proceso mediante un decreto supremo que convoque a un plebiscito de especial trascendencia, el que debería contar con el apoyo del Congreso.

Esta propuesta se ha articulado a través de la campaña denominada "plebiscito ahora”. Así, en la consulta se debería optar por las siguientes alternativas: (a) asamblea constituyente; (b) comisión bicameral (integrada por diputados y senadores que redactarían una propuesta de modificación constitucional), y (c) comisión de expertos nombrados por el Poder Ejecutivo con acuerdo del Poder Legislativo. En este último caso, dicha comisión trabajaría durante un tiempo acotado con el encargo exclusivo de redactar una propuesta de texto constitucional, la que deberá ser ratificada por los ciudadanos mediante un plebiscito ${ }^{24}$.

El punto es que mediante este plebiscito se vulneraría el procedimiento de reforma constitucional establecido en el capítulo XV de la actual Constitución, por lo que la intervención del Tribunal Constitucional según los procedimientos antes descritos resulta evidente.

24 Ver http://plebiscitoahora.cl/. 


\section{CONCLUSIÓN}

De nuestro análisis queda claro que la moción que pretende incorporar la figura del referéndum o plebiscito de especial trascendencia no tuvo a la vista la experiencia comparada en la materia. Ésta supone reservar este mecanismo para las potestades del Presidente de la República y en algunos casos se extiende al ámbito legal, pero sistemáticamente se aparta esta fórmula para los temas constitucionales. Incluso, nos encontramos con cartas fundamentales, como la colombiana, que llegan a prohibir expresamente el referéndum para establecer eventuales cambios en el proceso de reforma a la Constitución.

La experiencia comparada de alguna manera expresa algo que la doctrina también viene identificado desde hace algún tiempo: el referéndum no es una herramienta que promueva la participación y la deliberación constitucional. Más bien, tiende a ser vinculado con procesos cerrados, poco legitimados y que surgen mediante acuerdos de la élite política. Así, varios estudios cuantitativos que pretenden indagar en qué medida los procesos de elaboración de una nueva constitución impactan en la estabilidad del orden constitucional, identifican al referéndum como una herramienta de legitimación más que de participación ${ }^{25}$.

Así, el más reciente de estos estudios llega a señalar: "(...) hemos identificado un conjunto significativo de eventos en los que las constituciones fueron elaboradas por un organismo designado por la élite política de turno, posteriormente ratificada por un referéndum, y en el que el efecto de este proceso fue consolidar el control autoritario. Este hallazgo nos llevó a concluir que el uso de un referéndum no era necesariamente una característica de un proceso abierto y democrático" 26 .

Si miramos la experiencia latinoamericana, los procesos constituyentes que se iniciaron mediante plebiscitos muestran una polarización creciente que termina afectado el grado de deliberación de procesos supuestamente participativos. Un ejemplo paradigmático en este sentido es Venezuela, que mediante un referéndum realizado el 25 de abril de 1999 decidió la convocatoria a una asamblea constituyente, generando un nivel de beligerancia que hizo que la oposición se restara de toda esta discusión. Así, la abstención en el referéndum superó el 62\% y el $95.41 \%$ de representantes que resultaron electos pertenecían a la coalición gobernante ${ }^{27}$.

25 Widner (2008), Elkins et al., (2009) y WheAtley y Méndez (2013).

26 Wheatley y Méndez (2013), p. 434.

27 Hernández (2011), p. 231, y Renfer (2010), p. 325. 
De igual manera, estos efectos polarizadores en el debate constitucional se pueden observar en las consultas realizadas en Bolivia y Ecuador ${ }^{28}$.

Desde nuestra perspectiva, el proceso constituyente chileno debe entregar garantías de inclusión a todos los sectores políticos y esto supone articular una fórmula que permita deliberación y un proceso de remplazo constitucional. En ese sentido, se deben tomar en cuenta la experiencias comparadas exitosas, que ponen el acento más que en el órgano encargado de elaborar la nueva constitución, en el establecimiento de mecanismos de participación que generen actores crecientemente empoderados y que permitan dotar de legitimidad y estabilidad al nuevo orden constitucional que emerge de este debate. Esto lo podemos identificar en proyectos elaborados mediante asamblea constituyente, siendo el caso más destacado en la región el de Colombia ${ }^{29}$, o mediante el Congreso, donde destaca la experiencia de Brasil ${ }^{30}$.

\section{Bibliografía CitADA}

Aguiar de Luque, Luis (2001): “Referéndum”, en Reyes, Manuel (coordinador): Temas básicos de derecho Constitucional, (Madrid Editorial Civitas), tomo I. Aragón Reyes, Manuel (1989): Constitución y democracia, (Madrid: Tecnos).

Cruz Villalón, Pedro (1980): "El referéndum consultivo como modelo de racionalización constitucional”, Revista de Estudios Politicos, (No 13), pp. 145-168. Bonavides, P. (2001): Teoría Constitucional da Democracia Participativa, (Brasil: PC Editorial Ltda.).

Cepeda, M. (1993): La constituyente por dentro: mitos y realidades. (Bogotá: Presidencia de la República, Consejería para el desarrollo de la Constitución).

Elkins, Z., Ginsburg, T. y Melton, J. (2009): “The Endurance of National Constitutions", (Cambridge: Cambridge University Press).

Equipo de Sociología de la Universidad Autónoma de Barcelona: "El Referéndum del 12 de marzo de 1986 sobre la permanencia de España en la OTAN y sus consecuencias en el sistema político", Revista Española de Derecho Político, (No 52, julio-agosto de 1986), pp. 183-215.

28 Sото (2014).

29 Cepeda (1993), De la Calle (2004) y Zuluaga (2008).

30 Bonavides (2001) y Figueiredo (2008). 
Figueiredo, M. (2008): “La Evolución Político-Constitucional de Brasil”, Revista de Estudios Constitucionales (Año 6, No 2), pp. 210-238.

García-Pelayo, M. (1984): Derecho constitucional comparado, Introducción de Manuel Aragón, (Madrid: Alianza).

Garretón, Manuel Antonio (2014): "Plebiscito para una Asamblea Constituyente", en Le Monde Diplomatique, Edición chilena, (año XIV, No 150, abril), p. 4.

GonzÁlez Rissoto, R. (2008): "La democracia directa en Uruguay", en Revista Digital de Derecho Electoral, (Tribunal Supremo de Elecciones, No 6, San José, Costa Rica), pp. 1-26.

HERNÁNDEZ, Lolymar (2011): El proceso constituyente venezolano de 1999. Una exhortación por la limitación del poder como razón de ser de la norma constitucional (Caracas: Editorial Académica Española).

Pérez Sola, Nicolás (1994): La regulación constitucional del referéndum, Secretariado de Publicaciones, (Granada: Universidad de Jaén).

Pizzorusso, Alessandro (1987). Curso de Derecho Comparado, (Barcelona: Editorial Ariel).

LÓPEZ GONZÁLEZ, José Luis (2006):"El referéndum consultivo sobre decisiones de especial trascendencia: reflexiones desde el principio democrático en relación al referéndum sobre la constitución europea", en Revista Española de Derecho Político, (No 65), pp. 233-256.

Luciani, M. (2008): "Il Referéndum. Questioni teoriche e dell'esperienza italiana”, en Revita Catalana de Dret Públic, (No 37, Barcelona: Generalitat de Catalunya, Escola d' Administració Pública de Catalunya,) pp. 157-182. (2005): La formazione delle leggi, tomo I, 2 Art. 75, Il referéndum aborogativo, Comentario della Constituzione fondato da G. Branca a continuato da A. Pizzorusso, (Roma-Bologna: Zanichelli Editore Bologna, Il Foro Italiano). Olivetti, M. (2007): "Los referendos en serio: la experiencia italiana", en Revista Jurídica de la Universidad Autónoma de Madrid, (No 15), pp. 157-175.

Renfer, Irene (2010): Democracia directa en Argentina, Uruguay y Venezuela, tres ejemplos de democracia directa en América Latina, (Buenos Aires: Prometeo Libros).

SARTORI, G. (1987): Teoría de la democracia. (México: El debate contemporáneo, Alianza).

Schneider, Hans Peter (1991): Democracia y constitución, (Madrid: Centro de Estudios Constitucionales). 
SChumpeter, J. (1984): Capitalismo, socialismo y democracia, (Barcelona: Folio). Silva Bascuñán, Alejandro (1997): Tratado de Derecho Constitucional, Estado de excepción constitucional, gobierno y administración interior del Estado, reforma del Estado, (Editorial Jurídica de Chile), tomo X.

Soto Barrientos, Francisco (2014): "Asamblea Constituyente: la experiencia latinoamericana y el actual debate en Chile", en Revista Estudios Constitucionales, (Año 12, No 1), pp. 397-428.

(2013) El desafío de la participación: Estudio comparado sobre el referéndum y la iniciativa legislativa popular en América Latina y Europa, Santiago: LOM).

Welp, Yanina (2009): "España frente el referéndum europeo: o de la democracia y sus debilidades", Revista Confines, (Vol. 5, Núm. 10), pp. 39-53.

Wheatley J., Mendez F. (ed.) (2013): Patterns of Constitutional Design, The Role of Citizens and Elites in Constitution-Making, (England: Ashgate Publishing Limited).

WidNER, J. (2008): "Constitution writing in post-conflict settings: an overview". William \& Mary Law Review (No 49), pp. 1513-1540. 